\title{
Omitted medications: a continuing problem
}

\author{
Authors: Saurabh Shandilya, ${ }^{A}$ Khwaja Nizamuddin, ${ }^{\mathrm{B}}$ Mohammad Waqar Faisal, ${ }^{\mathrm{C}}$ Sajjad Noor ${ }^{\mathrm{D}}$ and Sam Abraham ${ }^{\mathrm{E}}$
}

Failure to provide prescribed medicines to inpatients has the potential to cause significant patient harm, to delay the resolution of the pathology and to increase the length of hospital stay. We measured the frequency at which medications were omitted in the non-admitting medical wards of a district general hospital, using two point-prevalence studies spaced one month apart. The results showed that the omission of prescribed medications remains a problem throughout the hospital stay of the patient. Among the charts studied, $73 \%$ had omitted medications. The most common cause of omission was patients' refusal (47.22\%), followed by patients' inability to take the medicine (22.7\%). Medication unavailability came third (17.04\%). Increased communication between medical, nursing and pharmacy staff, along with regular review of the patients by the patient team, with a view of reducing medication omission will go a long way in reducing the incidence of this problem.

KEYWORDS: Omitted medications, hospital admission, medication errors, hospital prescriptions, medicine reconciliation

\section{Introduction}

Patients who, for any reason, are admitted to the medicine department of a hospital can expect to be prescribed tablets, pills or intravenous (IV) medications. Many of these admissions are due to the worsening of preexisting conditions, and thus any improvement in the patient's clinical outcome is dependent on the continued administration of their previously prescribed medicines, along with new drugs or measures instituted on admission. The average number of medications being taken by a patient before they are admitted has been rising over the years. ${ }^{1}$ In this situation, it is essential that the treatments prescribed are administered as intended. Failure to do so has the potential to lead to patient harm. ${ }^{2}$ Furthermore, poor adherence with medication is often linked to decreased therapeutic effect and increased length of stay.

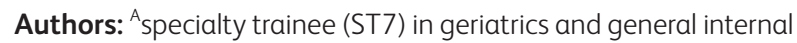
medicine, Wrexham Maelor Hospital, Wrexham, UK; ${ }^{B}$ core medical trainee (CT1), Wrexham Maelor Hospital, Wrexham, UK; ${ }^{C}$ core medical trainee (CT1), Bradford Royal Infirmary, Bradford, UK; ${ }^{\mathrm{D}}$ core medical trainee (CT1), Wrexham Maelor Hospital, Wrexham, UK; ${ }^{\text {E }}$ consultant geriatrician, Wrexham Maelor Hospital, Wrexham, UK
The first step in the prescription of a new patient's medicines is obtaining a good medication history, and this is a potential area of error. Physician-acquired medication histories have been shown to be inaccurate in up to $67 \%$ of cases and it has been noted that $27 \%$ of all hospital prescribing errors can be attributed to incomplete medication histories at admission. ${ }^{3}$ Improved physician training, accessible community pharmacy databases and closer teamwork between patients, physicians and pharmacists could reduce the frequency of these errors. ${ }^{4}$ The National Institute for Health and Care Excellence (NICE) and the National Patient Safety Agency (NPSA) recommend that medicine reconciliation with the help of pharmacists should be part of the admission process. ${ }^{5}$

The first few days of in-hospital care are probably the most important in a patient's recovery, and any medication omissions during this period have the potential to cause harm or to increase the necessary length of stay. An audit reported in 2009 by Green $e t a l^{6}$ found that $20 \%$ of the new prescriptions affecting $17 \%$ of the patients failed be administered in the first 48 hours of admission. Among the medications that were omitted, $7 \%$ were highly relevant medications that were omitted despite having been prescribed by the admitting doctors. Green et al $l^{6}$ found that in a hospital that was not using electronic prescribing, the most common cause of omission of prescribed medication in the first 48 hours of admission was that the medicine was unavailable on the ward; the second most common cause was the patient's being designated nil by mouth (NBM).

It has been our experience that the omission of prescribed medication is not confined to the admission wards. The administration of prescriptions for patients who require intermediate to long-term admissions in acute medical wards is also subject to various pressures, leading to their omission. These omissions are separate to administration errors. To look into this aspect of patient care, our team undertook an audit of the administration of prescribed medications in acute medical wards in a district general hospital.

\section{Method}

The prescription charts of patients admitted into acute medical (not admission) wards were studied on two different occasions. The data-collection exercise was carried out by the authors on three mid-week days in the month of April 2013. We looked at the prescription charts of all the inpatients on medical wards. Medical outliers on non-medical wards and the occasional direct admission patient on the medical wards were excluded. This exercise was assisted by the ward pharmacists and the nursing staff. The data-collection process was repeated in the month of 
Table 1. Reasons for omission of medications according to the all Wales prescription charts.

$\begin{array}{ll}\text { Code } & \text { Reason for omission } \\ 1 & \text { Prescriber's request } \\ 2 & \text { Patient not on ward } \\ 3 & \text { Patient unable to receive medicine or no access } \\ 4 & \text { Patient refused medicine } \\ 5 & \text { Medicine unavailable } \\ 6 & \text { See notes }\end{array}$

October on the same wards with the same caveats. The aim on both occasions was to look at 100 charts and we managed to get data from 96 charts in April and 104 in October. We chose midweek days and concentrated on medical wards so as to remove the confounding effects of low staffing levels on weekends and different nursing levels on the non-medical wards in which medical outliers were located. The accepted practice for All Wales prescription charts is for the nursing staff to record the reason for omission of medications under various categories (Table 1).

Using an audit sheet, which was blinded to the identification of the patients, we noted the demographics details along with the number of missed medications on the charts and the reasons given for their omissions. The data were then analysed, quantified and classified according to the types of medication, as determined according to the British National Formulary (BNF). The clinical relevance of the implicated medications was decided by the lead author (a medical registrar) who looked for omissions of medications such as antibiotics, heart-failure medications, antiParkinson medications and others mentioned in the NPSA alert.

\section{Results}

In total, 200 patients in acute medical wards were included in the study. The median age of the patients was 79 years (range 26-101 years); $45 \%$ were male. Of all the drug charts analysed, $73 \%$ had at least one omitted medication, while $48 \%$ had two or more medications omitted for various reasons. Only $27 \%$ of the charts showed full compliance with all the prescribed medications being administered.

A total of 456 instances of missed medications were found in the 200 charts studied and, as shown in Fig 1, the most common

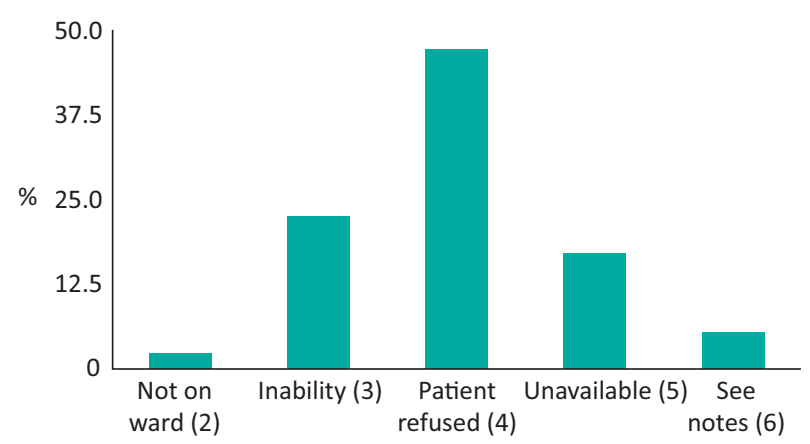

Fig 1. Reasons for omission of medications in percentages (code on drug chart). cause of missed medication was the refusal of the patient to take the medicine. This was seen in $217(47.22 \%)$ of the cases. The second most common reason was inability of the patient to receive the medication. This was the case in 103 (22.7\%) of the cases. Unavailability of the medications on the ward was the third most common cause (17.04\%), even though our study group was comprised of patients who had been in the hospital for more than 24 hours.

Many of the missed doses $(16.83 \%)$ were considered to be highly relevant. These included one case of a patient who was admitted with sepsis but had missed his doses of co-amoxiclav because of unavailability.

Classification of the omitted medications according to the BNF revealed that the missed medications were for the treatment of various disease subgroups (Table 2).

\section{Discussion}

Failure to administer medications is not an uncommon problem and accounts for the second largest cause of medication incidents reported in the UK. ${ }^{7}$ Among the 5,437,999 medication incidents reported across the NHS between 2005 and 2010, $15.6 \%$ resulted from missed or delayed medication. ${ }^{8}$ This has led to the inclusion of medicine-management issues in many local and regional targets following the introduction of the Commissioning for Quality and Innovation agenda in 2009. ${ }^{9}$

In our study, we established that the problem of omission of medications is not limited to the initial prescription or the first few days of admission but is a continuing problem. The most common cause of missed medications in our experience was patients refusing medications (47\%), followed by inability of the patient to receive the medication (22\%) either because they were NBM and thus unable to take oral medications or because there was no IV access for IV medications. Medication unavailability, which was the dominant cause of omission of medications in admission wards, was responsible for only $17 \%$ of the omissions for medical inpatients.

When a patient refuses to take their prescribed medication, it is important to find out why. First, we must ascertain that the patient has the capacity to make this decision. In an

Table 2. Incidence of omitted medications classified according to BNF.

\begin{tabular}{ll} 
Category & Percentage \\
Gastroenterology & 17.5 \\
Cardiology & 16.8 \\
Respiratory & 7.7 \\
CNS & 15.6 \\
Anti-infectives & 7.2 \\
Endocrinology & 8.3 \\
Nutrition & 10.3 \\
Musculoskeletal & 12.7 \\
Eye & 2.0 \\
Dermatology & 1.7 \\
\hline BNF = British National Formulary; CNS = central nervous system.
\end{tabular}


increasingly geriatric inpatient population, the clouding of consciousness resulting from an acute delirium caused by the admitting diagnosis is not uncommon. If the reason for refusal is confusion on the part of the patient, every effort should be made to explain the need for the medication. If these efforts are unsuccessful, alternative means of drug delivery such as IV or subcutaneous routes may be used. In some selected cases, covert administration of medication according to the local protocols may be needed.

In patients who are not confused, the causes for refusal of medications need to be looked at on an individual level. In our study population, we found that the most common type of medications that were missed were analgesics, followed by laxatives. It was also noted that there were repetitive refusals of the same medications in the same chart, leading to the conclusion that these prescriptions would have been better suited to the as-and-when-needed (PRN) side of the medication chart rather than the regular-dose side. We suggest that if the medical or nursing and pharmacy staff are conscious of this issue, it will reduce the incidence of omissions of medications and reduce the time spent by the pharmacy staff in dispensing, nursing staff in administering and medical staff in reviewing the medications.

The inability to administer medications because of lack of an available route of administration was the cause of 103 medication omissions. The two main reasons for the omission of an oral medication were that the patient was either NBM or drowsy. It should be part of medical practice that whenever a patient is made NBM, an immediate review of the medication chart should be carried out to prescribe alternate routes of administration for the necessary medication (with provision of alternative medications if needed) and for planned withholding of the non-essential oral medications. This will ensure that the patient's recovery is not jeopardized by their inability to swallow safely. It was noted that many of the decisions to stop the oral feeding of patients were made by the on-call medical teams out of hours. Given the paucity of medical staff at such times and the pressures on the on-call team, it is difficult to propose measures that will increase their already high work load. As a minimum, the ward-based medical teams should review the medications for any patient made NBM in the outof-hours period at the earliest opportunity, and this must be communicated to the ward pharmacists and nursing staff.

If the reason for not giving the medication was that the patient was drowsy then the cause for their drowsiness must be looked into and appropriate solutions found.

The absence of intravenous access for administering of IV medications was also a recurring theme in our study. The provision of more ward nurses with competencies to establish IV access should be aimed for. The dependence on the medical team, especially the on-call team, causes delays in medicine delivery, which when antibiotics, anti-epileptic medications, diuretics or proton pump inhibitors are needed might have rapidly deleterious effects on the health of the patient.

The number of patients who could not receive their prescribed medications because the drug was not available on the ward was $17.0 \%$. This was despite the fact that all of the patients whose charts were included in the study had been in the hospital for more than 24 hours, and thus there had been ample opportunity for the medical and pharmacy team to arrange for these medications to be available.
Discussions with pharmacy teams involved in procuring the medication for ward use revealed that if the medical team had prescribed medications on the drug chart after the daily pharmacists' round and had not highlighted this to the nursing staff, then any drugs that are not readily available on the ward will be ordered only after the next medication round. This delay creates the potential for the patient to miss two to three doses of the newly prescribed medication.

We suggest that increased communication between medical, nursing and pharmacy teams is essential for ensuring that the prescribed medications reach their intended targets. When medications are prescribed or altered after the pharmacy rounds, it is important that the prescribing doctor communicates this information to the nursing staff, so that appropriate measures are taken to order the necessary drugs.

In summary, the issues around omission of medications remain an area of concern throughout the inpatient stay. The cause for missing medications in intermediate and long-stay patients differ from those affecting short stay and newly admitted patients. These issues require the continued attention of the treating medical teams and robust lines of communication between medical, nursing and pharmacy teams.

\section{References}

1 Gorard D, Kind A. Prescribing patterns in acutely admitted medical patients: changes over 10 years. Clin Med 2005;5:663-4.

2 Ferner RE, Aronson J. Clarification of terminology in medication errors: definitions and classification. Drug Saf 2006;29:1011-22.

3 Dobrazanski S, Hammond I, Khan G, Holdsworth H. The nature of hospital prescribing errors. Br J Clin Govern 2002;7:187-93.

4 Tam VC, Knowles SR, Cornish PL et al. Frequency, type and clinical importance of medication history errors at admission to hospital: a systematic review. CMAJ 2005;173;510-5.

5 National Institute for Clinical Excellence/National Patient Safety Agency. Technical patient safety solutions for medicines reconciliation on admission of adults to hospital. (Alert reference: NICE/ NPSA/2007/PSG001). London: NHS, 2007. Available online at www. nice.org.uk/guidance/psg001/resources/full-guidance-pdf2 [Accessed 1 December 2014].

6 Green CJ, Du-Pre P, Elahi N, Dunckley P, McIntyre AS. Omission after admission: failure in prescribed medications being given to inpatients. Clin Med 2009;9:515-8.

7 National Patient Safety Agency. Safety in doses. Improving the use of medicines in the NHS, 2009. Available online at www.nrls.npsa.nhs. uk/resources/?entryid45=61625 [Accessed 1 December 2014].

8 Cousins DH, Gerrett D, Warmer B. A review of medication incidents reported to the National reporting and Learning System in England and Wales over 6 years (2005-2010). Br J Clin Pharmacol 2012;74:597-604.

9 Darzi A. High quality care for all: NHS next stage review (final report). London: DH, 2008. Available online at http://webarchive. nationalarchives.gov.uk/20130107105354/http://www.dh.gov.uk/en/ Publicationsandstatistics/Publications/PublicationsPolicyAnd Guidance/DH_085825 [Accessed 1 December 2014].

Address for correspondence: Dr S Shandilya, Wrexham Maelor Hospital, Care of Elderly Department, Croesnewydd Road, Wrexham LL13 7TD, UK. Email: drshandilya@gmail.com 